\title{
Avaliação da microcirculação das bordas do tendão do supra-espinal nas lesões do manguito rotador
}

\author{
Microvascular evaluation of the supraspinatus \\ tendon borders in rotator cuff lesions
}

\author{
Roberto Yukio IKEMOto', Joel MurachovskY², LUIS GustaVo Prata Nascimento³, \\ RogérIo SERPONe BUENO ${ }^{4}$, FABRícIO HIDETOSHI UENO ${ }^{5}$, JORge AKITA JÚNIOR ${ }^{5}$
}

\section{RESUMO}

Objetivos: Avaliar a microcirculação das bordas do tendão supra-espinal nas lesões do manguito rotador com a finalidade de determinar a necessidade ou não do desbridamento de suas bordas no momento do seu reparo cirúrgico. Métodos: No período de junho a dezembro de 2004 , foram avaliadas amostras recolhidas de 31 pacientes portadores de lesão completa do tendão supra-espinal, submetidos ao tratamento da lesão do manguito rotador por via artroscópica. Apresentavam idade entre 42 e 82 anos (média de 56,6 anos), sendo nove do sexo masculino e 22 do feminino. Durante a realização do procedimento, foram retiradas amostras de tecido da lesão do manguito rotador e enviadas

* Trabalho realizado pela Disciplina de Doenças do Aparelho Locomotor da Faculdade de Medicina do ABC, Santo André (SP), Brasil (Serviço do Prof. Dr. Carlo Milani).

1. Mestre; Chefe do Grupo de Ombro e Cotovelo da Faculdade de Medicina do ABC, Santo André (SP), Brasil.

2. Doutor; Médico Assistente do Grupo de Ombro e Cotovelo da Faculdade de Medicina do ABC, Santo André (SP), Brasil.

3. Médico Assistente do Grupo de Ombro e Cotovelo da Faculdade de Medicina do ABC, Santo André (SP), Brasil.

4. Médico Assistente do Grupo de Ombro e Cotovelo da Faculdade de Medicina do ABC, Santo André (SP), Brasil.

5. Residente do 3ํ Ano de Ortopedia e Traumatologia da Faculdade de Medicina do ABC, Santo André (SP), Brasil.

Endereço para correspondência: Rua Monte Alegre, 253-121, Santo Antônio - 09531-110 - São Caetano do Sul, SP. Tel.: 4229-0852.

E-mail: rehema@terra.com.br

Recebido em 23/6/07. Aprovado para publicação em 10/12/07.

Copyright RBO2007 para estudo anatomopatológico com coloração com hematoxilina-eosina. Após esse processo, foi realizada a contagem das fendas vasculares $/ \mathrm{mm}^{2}$. Utilizaram-se como grupo controle 10 amostras de tendões normais do supra-espinal de cadáveres frescos, submetidos aos mesmos processos anteriores. Os resultados obtidos foram avaliados estatisticamente através da aplicação do teste de Mann-Whitney. $R e$ sultados: Entre as amostras, 28 apresentaram tecidos vascularizados e três, ausência de vascularização. O número médio de fendas vasculares $/ \mathrm{mm}^{2}$ nas amostras de lesões do manguito rotador foi estatisticamente maior que o do grupo controle. Conclusão: A maioria das bordas das lesões dos tendões do supra-espinal é hipervascularizada.

Descritores - Bainha rotadora/lesões; Bainha rotadora/cirurgia; Microcirculação; Artroscopia

\section{ABSTRACT}

Objectives: To evaluate microvasculature in the borders of the supraspinatus tendon in rotator cuff lesions in order to determine the need to debrid the borders when surgical repair is performed. Methods: From June to December 224, samples were evaluated from 31 patients with full lesion of the supraspinatus tendon that had been submitted to arthroscopic rotator cuff lesion treatment. They were between 42 and 82 years of age (mean 56.6 years), nine of them male, and twenty-two female. During the procedure, samples of the rotator cuff lesion tissue were collected and sent to the anatomopathological study with 
hematoxylin-eosin dye. Afterwards, a counting of the vascular slits $/ \mathrm{mm}^{2}$ was done. The authors used as a control group ten samples of normal supraspinatus tendons from fresh cadavers that were submitted to the same procedures. Results obtained were statistically evaluated by the Mann-Whitney test. Results: Of all samples, 28 presented vascularized tissues and three were not vascularized. The mean number of vascular slits $/ \mathrm{mm}^{2}$ in rotator cuff lesion samples was statistically higher than that of the control group. Conclusion: Most borders of supraspinatus tendon lesions are hypervascular.

Keywords - Rotator cuff/injuries; Rotator cuff/surgery; Microcirculation; Arthroscopy

\section{INTRODUÇÃO}

O tendão mais freqüentemente acometido nas lesões do manguito rotador é o do músculo supra-espinal e a causa dessa lesão é motivo de controvérsia ${ }^{(1)}$. Hipovascularização, degeneração e trauma são fatores apontados como responsáveis por essa lesão.

O padrão vascular do manguito rotador é apontado como um dos principais fatores dessa lesão. Codman descreveu uma região do tendão do supra-espinal denominada de "área crítica", local onde freqüentemente ocorre rotura $^{(2)}$. Essa região corresponde à anastomose entre os vasos ósseos e tendinosos do supra-espi$\mathrm{nal}^{(3)}$. A partir desse estudo, diversos autores passaram a avaliar a vascularização do manguito rotador.

Moseley et al concluíram que o padrão vascular dos tendões do manguito rotador é o mesmo em todas as faixas etárias e que os vasos se tornam mais tortuosos e finos com o avançar da idade; eles não identificaram ser a zona crítica menos vascularizada que outras áreas do manguito rotador ${ }^{(3)}$. Rothman et al postularam ser a zona crítica hipovascularizada associada ao avanço da idade e não um estado patológico ${ }^{(4)}$.

Rathbun et al propuseram o desbridamento das bordas da lesão do manguito rotador nos casos em que havia hipovascularização do tendão do supra-espinal e suas conseqüentes alterações degenerativas e, nesse estudo, concluíram que a maioria dos tendões nas lesões completas do supra-espinal era avascular, defen- dendo, portanto, a excisão da parte degenerada de suas bordas $^{(5)}$. Desde então, alguns autores vêm defendendo o desbridamento das lesões, porém seus estudos são baseados na vascularização em cadáveres ${ }^{(6-7)}$. Existem autores que afirmam não haver necessidade de tal desbridamento, baseados em análises histológicas de biópsias cirúrgicas de lesões do manguito rotador que revelaram proliferação vascular nas suas bordas ${ }^{(8-10)}$.

Devido a essa controvérsia, realizamos este estudo com o objetivo de avaliar o padrão microvascular das bordas das lesões do tendão supra-espinal durante seu reparo cirúrgico artroscópico.

\section{MÉTODOS}

Entre junho e dezembro de 2004, o Grupo de Ombro e Cotovelo do Departamento de Ortopedia e Traumatologia da Faculdade de Medicina do ABC tratou 31 pacientes portadores de lesão completa do tendão do supra-espinal por meio do reparo artroscópico. Todos foram submetidos ao estudo do padrão vascular das bordas das lesões do tendão sob aprovação do Comitê de Ética da Instituição. A média de idade foi de 55,6 anos, variando de 42 a 82 anos. Entre os pacientes, nove eram do sexo masculino e 22 do feminino.

Com relação ao mecanismo de lesão, em 20 casos havia relato de trauma e em 11, não. Durante o reparo artroscópico foi realizada biópsia do tendão, de 5 a $10 \mathrm{~mm}$ proximais à borda do supra-espinal e, em seguida, o material foi encaminhado para estudo anatomopatológico para avaliação da microcirculação local. Tais amostras foram armazenadas em formalina a $10 \%$, submetidas a banhos de álcool absoluto e preparo com xilol e parafina aquecida a $60^{\circ} \mathrm{C}$. Foi empregada parafina histológica com cortes finos (cinco micrômetros) e coloração por hematoxilina-eosina. Após todo o processo, realizou-se então a contagem do número de fendas vasculares por $\mathrm{mm}^{2}$.

Selecionamos como grupo controle 10 amostras de tendões normais do supra-espinal de cadáveres frescos (coletados no S.V.O. de Santo André); o local para análise foi a borda na inserção do tendão. A média de idade dos cadáveres era de 67,9 anos (variando de 35 a 87 anos), sendo nove do sexo masculino e um do feminino. Todos foram submetidos aos mesmos pro- 

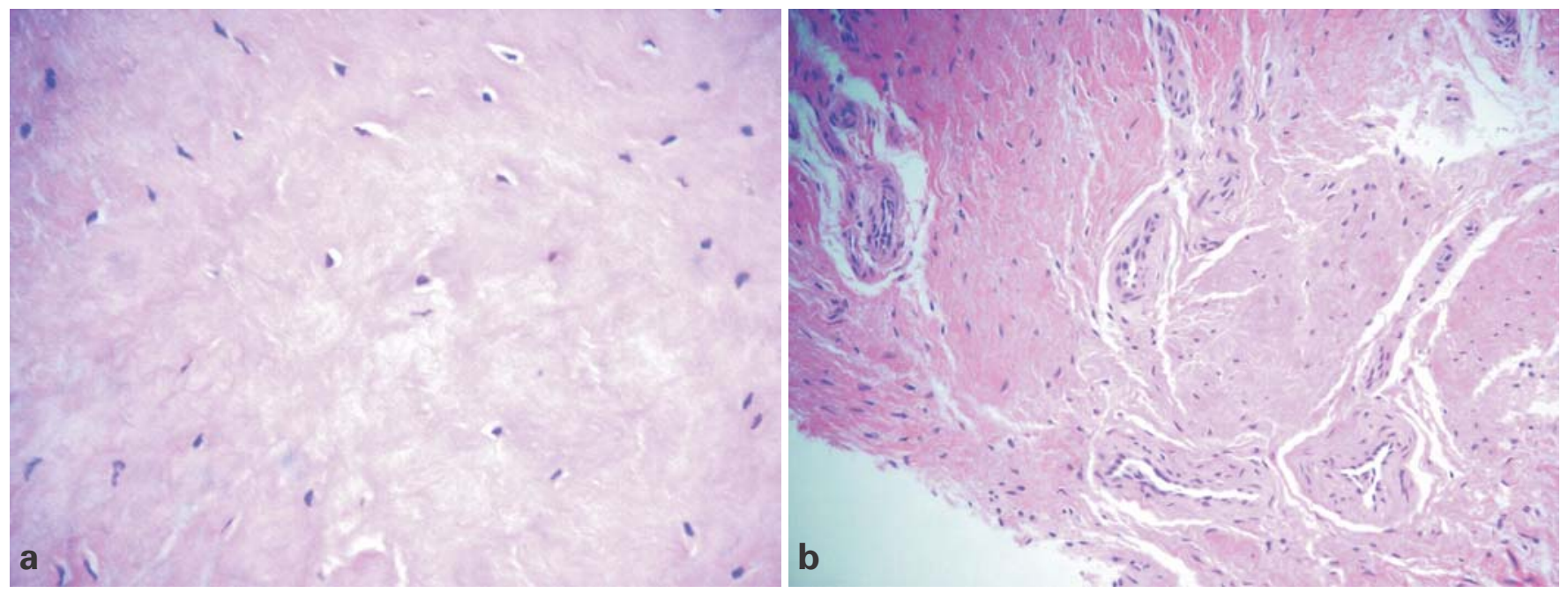

Figura 1 - Amostras de tecido armazenadas em formalina a 10\%, submetidas a banhos de álcool absoluto, preparados com xilol e parafina aquecida a $60^{\circ} \mathrm{C}$. Parafina histológica com cortes finos (cinco micrômetros) e coloração por hematoxilina-eosina: (a) tecido avascularizado (x400); (b) tecido vascularizado (x40).

cessos de preparo e estudo da vascularização, para depois compará-los com o padrão vascular obtido nas amostras das lesões dos pacientes.

Os resultados obtidos foram analisados estatisticamente por meio da aplicação do teste de Mann-Whitney, com o intuito de verificar possíveis diferenças de valores das variáveis de interesse.

\section{RESULTADOS}

Das 31 amostras coletadas e enviadas para o estudo anatomopatológico, 28 (90,3\%) apresentavam tecido vascularizado (figura 1a) e três $(9,7 \%)$, ausência de vascularização (figura 1b). No primeiro grupo, o tempo médio de dor foi de 31,6 meses e no segundo, de 16,7 meses.

A área média das amostras das lesões foi de $10,3 \mathrm{~mm}^{2}$ (variando de $3 \mathrm{a} 19 \mathrm{~mm}^{2}$ ) e o número médio de fendas vasculares $/ \mathrm{mm}^{2}$ foi 1,56 (variando de 0 a 3,88 ). O grupo controle teve área média de $24,4 \mathrm{~mm}^{2}$ (variando de $10 \mathrm{a} 40 \mathrm{~mm}^{2}$ ) e o número médio de fendas vasculares/ $\mathrm{mm}^{2}$ de 0,24 (variando de 0,075 a $0,6 \mathrm{~mm}^{2}$ ) (gráficos 1 e 2).

Portanto, de acordo com o teste de Mann-Whitney, o número de fendas $/ \mathrm{mm}^{2}$ do grupo das lesões do manguito rotador, submetidas à biópsia no ato cirúrgico, foi estatisticamente maior do que o número de fendas vasculares encontradas no grupo controle (tabela 1).
A vascularização do tecido não teve correlação com a idade, sexo e nem com o tempo de dor encontrados em ambos os grupos.

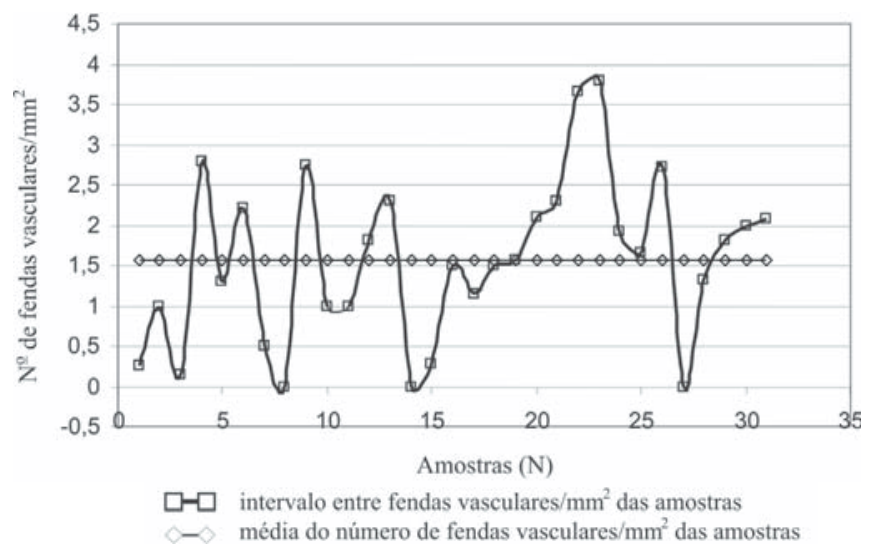

Gráfico 1 - Número de fendas vasculares $/ \mathrm{mm}^{2}$ no grupo de lesões do tendão do manguito rotador. Variação: $0 / \mathrm{mm}^{2}-3,88 / \mathrm{mm}^{2}$. Média: $1,56 / \mathrm{mm}^{2}$.

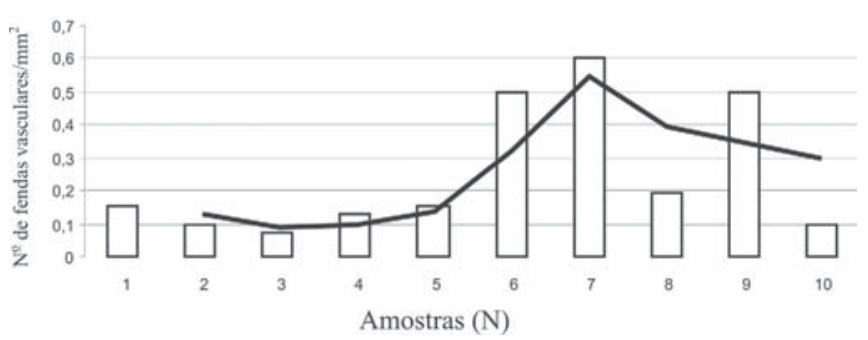

Gráfico 2 - Número de fendas vasculares $/ \mathrm{mm}^{2}$ do grupo controle. Média $=0,24 / \mathrm{mm}^{2}$. Variação $=0,075-0,6 / \mathrm{mm}^{2}$. 
TABELA 1

Análise estatística da vascularização $/ \mathrm{mm}^{2}$ das amostras das lesões do manguito rotador $x$ grupo controle

\begin{tabular}{llllcc}
\hline \multicolumn{1}{c}{ Variável } & Grupo & N & Média & DP & P \\
\hline Área $/ \mathrm{mm}^{2}$ & $\begin{array}{l}\text { Lesões do } \\
\text { das amostras }\end{array}$ & 31 & 10,0323 & 3,8426 & $<0,001$ \\
& $\begin{array}{l}\text { Conguito } \\
\text { Controle }\end{array}$ & 10 & 24,4000 & 7,9889 & \\
\hline № de & Lesões do & 31 & 1,5619 & 1,0216 & 0,001 \\
fendas $/ \mathrm{mm}^{2}$ & $\begin{array}{l}\text { manguito } \\
\text { Controle }\end{array}$ & 10 & 0,2490 & 0,2006 & \\
& & & & & \\
\hline
\end{tabular}

(Teste de Mann-Whitney)

$\mathrm{N}$ = amostras; $\mathrm{DP}=$ desvio-padrão; $\mathrm{P}$ = significância

\section{DISCUSSÃO}

O tendão do músculo supra-espinal é o mais freqüentemente acometido nas lesões do manguito rotador, tornando-se de fundamental importância o conhecimento do padrão vascular dessas lesões em relação à sua patogenia e ao seu tratamento ${ }^{(1)}$.

Assim como na literatura, a vascularização do tecido não teve correlação com a idade, sexo e nem com o tempo de dor encontrado em ambos os grupos $^{(3,10)}$.

A maioria dos trabalhos que analisam a vascularização do manguito rotador é baseada em estudos realizados em cadáveres e mostrou resultados conflitantes ${ }^{(10)}$. Alguns autores recomendavam o desbridamento das bordas das lesões baseados na presença de hipovascularização na zona crítica descrita por $\operatorname{Codman}^{(1,}$ ${ }^{4-5)}$. Moseley et al não observaram esse padrão vascular em cadáveres ${ }^{(3)}$. Segundo Goodmurphy et al isso pode ocorrer devido a diferentes técnicas empregadas para a realização desses estudos ${ }^{(10)}$.

Obtivemos, na maioria dos casos, aumento da vascularização nas bordas das lesões do manguito rotador $(90,3 \%)$ semelhante aos resultados de outros estudos que analisam histologicamente biópsias cirúrgicas ${ }^{(8-10)}$. O mesmo também foi observado por Brooks et al que, por meio de laser-doppler-fluxometria, encontraram hipervascularização nas bordas das lesões, sugerindo assim que o tecido adjacente à lesão não necessitaria de desbridamento ${ }^{(11)}$.

Acredita-se que essa hipervascularização encontrada nas bordas das lesões do manguito rotador seja uma resposta inflamatória do tipo reação de corpo estranho às mudanças degenerativas progressivas do tendão ${ }^{(3,5)}$.

A cicatrização do tecido não depende somente da vascularização, mas também de sua capacidade de produzir colágeno, que agirá como suporte da matriz extracelular. Sano et al confirmaram esse fato, demonstrando que o afilamento e a irregularidade das fibras colágenas e a presença de tecido de granulação predispõem à falha na cicatrização do tendão no seu sítio de inserção ${ }^{(12)}$. Goodmurphy et al realizaram análises imunocitoquímicas de biópsias cirúrgicas de lesões do manguito rotador e demonstraram um padrão de vascularização sem alteração na área compreendida da borda da lesão do tendão até $2,5 \mathrm{~mm}$ proximais, comparado com seu grupo controle, e hipervascularização na área de 2,5 a $5,0 \mathrm{~mm}$ proximais à borda da lesão ${ }^{(10)}$. Além disso, evidenciaram, assim como outros autores, a capacidade de síntese de procolágeno tipo I, precursor desse tipo de colágeno, que é o maior componente presente nos tendões ${ }^{(9-10)}$.

Em decorrência das alterações degenerativas do tendão - irregularidade das fibras colágenas, infiltração gordurosa, degeneração mixóide e hialina, entre outras, mesmo não havendo hipovascularização significativa - justifica-se o desbridamento artroscópico dessa área, procedimento fácil e rápido e que possivelmente viabiliza a cicatrização do tendão(12-13).

\section{CONCLUSÃO}

As bordas das lesões do tendão do músculo do supra-espinal são hipervascularizadas na sua maioria, mas existe uma percentagem menor $(9,7 \%)$ avascular.

\section{REFERÊNCIAS}

1. Lohr JF, Uhthoff HK. The microvascular pattern of the supraspinatus tendon. Clin Orthop Relat Res. 1990;(254):35-8.

2. Codman EA. The shoulder. Boston: Thomas Todd; 1934. p. 65107.

3. Moseley HF, Goldie I. The arterial pattern of the rotator cuff of the shoulder. J Bone Joint Surg Br. 1963;45:780-9.

4. Rothman RH, Parke WW. The vascular anatomy of the rotator cuff. Clin Orthop Relat Res. 1965;41:176-86.

5. Rathbun JB, Macnab I. The microvascular pattern of the rotator cuff. J Bone Joint Surg Br. 1970;52(3):540-53. 
6. Hawkins RH. The rotator cuff and biceps tendon. In: Evarts M, editor. Surgery of the musculoskeletal system. New York: Churchill Livinsgtone; 1983. p. 128-32.

7. Craig E. Open anterior acromioplasty for full thickness rotator cuff tears. In: Craig EV, editor. The shoulder. New York: Raven Press; 1995. p. 21-7.

8. Uhthoff HK, Kumagai J, Sarkar K, Lohr J, Murnaghan P. Morphologic evidence of healing in torn human rotator cuffs. $\mathrm{J}$ Bone Joint Surg Br. 1992;74(Suppl 3):293-4.

9. Fukuda H, Hamada K, Yamanaka K. Pathology and pathogenesis of bursal-side rotator cuff tears viewed from en bloc histologic sections. Clin Orthop Relat Res. 1990;(254): 75-80.
10. Goodmurphy CW, Osborn J, Akesson EJ, Johnson S, Stanescu V, Regan WD. An immunocytochemical analysis of torn rotator cuff tendon taken at time of repair. J Shoulder Elbow Surg. 2003;12(4):368-74.

11. Brooks CH, Revell WJ, Heatley FW. A quantitative histological study of the vascularity of the rotator cuff tendon. J Bone Joint Surg Br. 1992;74(1):151-3.

12. Sano H, Ishii H, Trudel G, Uhthoff HK. Histologic evidence of the degeneration at the insertion of 3 rotator cuff tendons: a comparative study with human cadaveric shoulders. J Shoulder Elbow Surg. 1999;8(6):574-9.

13. Hashimoto T, Nobuhara K, Hamada T. Pathologic evidence of degeneration as a primary cause of rotator cuff tear. Clin Orthop Relat Res. 2003;(415):111-20. 